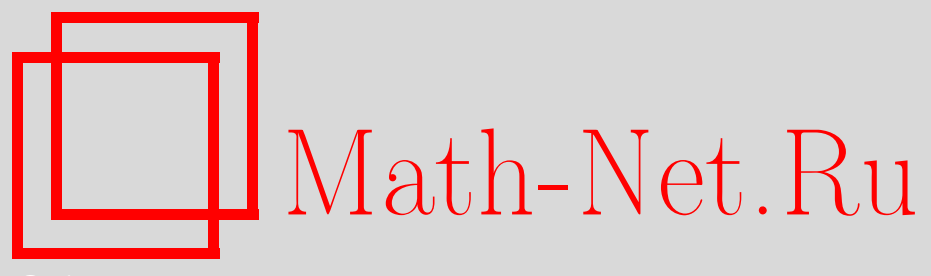

M. А. Скрябин, Модельная задача в конусе для функционально-дифференциального оператора с преобразованиями поворота, УМH, 2009, том 64, выпуск 1, 159-160

DOI: https://doi.org/10.4213/rm9274

Использование Общероссийского математического портала Math-Net.Ru подразумевает, что вы прочитали и согласны с пользовательским соглашением http://www . mathnet.ru/rus/agreement

Параметры загрузки:

IP: 3.81 .55 .215

26 апреля 2023 г., 16:00:35

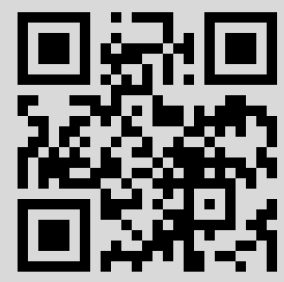




\section{Модельная задача в конусе для функционально-дифференциального оператора с преобразованиями поворота}

\section{М. А. Скрябин}

Необходимые и достаточные условия сильной эллиптичности в алгебраической форме для дифференциальных операторов были получены М. И. Вишиком и Л. Гордингом [1], [2]. Позднее А. Л. Скубачевский [3] получил необходимые и достаточные условия сильной эллиптичности для дифференциально-разностных операторов в виде положительной определенности некоторых матриц. Используя аппарат банаховых алгебр, Л. Е. Россовский [4] получил необходимые и достаточные условия сильной эллиптичности в алгебраической форме для функционально-дифференциальных операторов с постоянными коэффициентами с преобразованиями растяжения и сжатия аргумента. Полученные им условия записывались в виде положительности некоторой функции. В настоящей работе рассматриваются функционально-дифференциальные операторы с преобразованиями поворота.

Пусть $Q=\left\{x \in \mathbb{R}^{n}: x_{1}^{2}+\cdots+x_{n-1}^{2}=\alpha x_{n}^{2}\right\}$ - конус в $\mathbb{R}^{n}$. Рассмотрим функционально-дифференциальный оператор

$$
(A u)(x)=-\sum_{i, j=1}^{n}\left(\Phi_{i j} u_{x_{j}}\right)_{x_{i}}+\sum_{i=1}^{n} \Phi_{i} u_{x_{i}}+\Phi_{0} u(x),
$$

действующий из $\dot{C}^{\infty}(Q)$ в $L_{2}(Q)$. Здесь операторы $\Phi_{i j}: L_{2}(Q) \rightarrow L_{2}(Q), i, j=1, \ldots, n$, и $\Phi_{i}: L_{2}(Q) \rightarrow L_{2}(Q), i=0, \ldots, n$, являются линейными и ограниченными, причем

$$
\left(\Phi_{i j} u\right)(x)=\sum_{g \in G} a_{i j g} u(g(x)),
$$

где $G$ - конечное множество преобразований поворота относительно $0, a_{i j g} \in \mathbb{C}$. Так как, вообще говоря, $g(x) \notin Q$ при $x \in Q$, то при определении операторов $\Phi_{i j}$ предполагается, что $u(x) \equiv 0$ вне $\bar{Q}$.

Оператор $A$ называется сильно эллиптическим в $\bar{Q}$, если для всех $u \in \dot{C}^{\infty}(Q)$ справедливо неравенство

$$
\operatorname{Re}(A u, u)_{L_{2}(Q)} \geqslant c_{1}\|u\|_{W_{2}^{1}(Q)}^{2}-c_{2}\|u\|_{L_{2}(Q)}^{2},
$$

где константы $c_{1}>0, c_{2} \geqslant 0$ не зависят от функции $u(x)$. Здесь через $W_{2}^{1}(Q)$ обозначено пространство Соболева порядка 1.

Обозначим через $\mathscr{G}$ группу преобразований поворота относительно 0, порожденную конечным множеством $G$. Группа $\mathscr{G}$ не предполагается коммутативной, но предполагается конечной. Орбитой точки $x \in \bar{Q}$ (под действием группы $\mathscr{G}$ ) будем называть множество $\operatorname{orb} x=\{y: y=g(x), g \in \mathscr{G}\} \cap \bar{Q}$. Так как по предположению группа $\mathscr{G}$ конечна, то и все орбиты $\operatorname{orb} x$ конечны.

При исследовании сильной эллиптичности функционально-дифференциальных операторов наиболее сложным является случай неподвижной точки. В рассматриваемой нами модельной задаче не существенна геометрия области $Q$ (существенно то, что $0 \in \partial Q$ и что орбиты $\operatorname{orb} x$ конечны). Также несущественным является требование, чтобы $a_{i j g}$ были константами: в случае переменных коэффициентов их можно заморозить в точке 0 .

Пусть $\operatorname{orb} x_{0}=\left\{x_{0}, \ldots, x_{N}\right\}$ - орбита некоторой точки $x_{0} \in \bar{Q}$. Несложно показать, что существуют преобразования $g_{l} \in \mathscr{G}, l=0, \ldots, N$, определяющиеся единственным образом, такие, что $g_{l}\left(x_{0}\right)=x_{l}$ (очевидно, что $g_{0}(x)$ - тождественное преобразование).

Работа выполнена при поддержке РФФИ (грант № 07-01-00268). 
Для каждого оператора $\Phi_{i j}$ и множества orb $x_{0}=\left\{x_{0}, \ldots, x_{N}\right\}$ введем матрицу $\Phi_{i j, x_{0}}=\left\|\varphi_{k l}^{i j}\right\|_{k, l=0}^{N}$ порядка $(N+1) \times(N+1)$, элементы которой вычисляются по формуле

$$
\varphi_{k l}^{i j}= \begin{cases}a_{i j g}\left(g_{k}\left(x_{0}\right)\right), & \text { если } g=g_{k}^{-1} \circ g_{j} \in G, \\ 0, & \text { если } g \notin G .\end{cases}
$$

Кроме того, введем следующие матрицы размера $(N+1) \times(N+1)$ :

$$
\begin{aligned}
\frac{\partial\left(\mathbf{g}^{-1}\right)_{p}}{\partial x_{i}} & =\operatorname{diag}\left\{\frac{\partial\left(g_{0}^{-1}\right)_{p}}{\partial x_{i}}, \ldots, \frac{\partial\left(g_{N}^{-1}\right)_{p}}{\partial x_{i}}\right\} \quad(p=1, \ldots, n), \\
\Psi_{p q, x_{0}} & =\sum_{i, j=1}^{n} \frac{\partial\left(\mathbf{g}^{-1}\right)_{p}}{\partial x_{i}} \Phi_{i j, x_{0}} \frac{\partial\left(\mathbf{g}^{-1}\right)_{q}}{\partial x_{j}} \quad(p, q=1, \ldots, n),
\end{aligned}
$$

где $g_{l}^{-1}(x)=\left(\left(g_{l}^{-1}\right)_{1}(x), \ldots,\left(g_{l}^{-1}\right)_{n}(x)\right)^{T}, l=0, \ldots, N$.

Тогда необходимые и достаточные условия сильной эллиптичности оператора $A$ (см. (1)) можно сформулировать в виде следующей теоремы.

Теорема. 1) Пусть оператор А является сильно эллиптическим в $\bar{Q}$. Тогда для любой точки $x_{0} \in Q$ такой, что оrb $x_{0} \cap \partial Q=\varnothing$, и для любого вектора $\xi \in \mathbb{R}^{n}$, $\xi \neq 0$, матрищъ

$$
\sum_{p, q=1}^{n}\left(\Psi_{p q, x_{0}}+\Psi_{p q, x_{0}}^{*}\right) \xi_{p} \xi_{q}
$$

положительно определены.

2) Пусть для любой точки $x_{0} \in \bar{Q}$ и для любого вектора $\xi \in \mathbb{R}^{n}, \xi \neq 0$, матриць

$$
\sum_{p, q=1}^{n}\left(\Psi_{p q, x_{0}}+\Psi_{p q, x_{0}}^{*}\right) \xi_{p} \xi_{q}
$$

положительно определены. Тогда оператор А является сильно эллиптическим в $\bar{Q}$.

Автор выражает глубокую благодарность А. Л. Скубачевскому за внимание к работе.

\section{Список литературы}

[1] М. И. Вишик, Матем. сб., 29(71):3 (1951), 615-676. [2] L. Gårding, Math. Scand., 1 (1953), 55-72. [3] A. L. Skubachevskii, Elliptic functional differential equations and applications, Oper. Theory Adv. Appl., 91, Birkhäuser, Basel, 1997. [4] Л. Е. Россовский, Матем. заметки, 59:1 (1996), 103-113; англ. пер.: L. E. Rossovskii, Math. Notes, 59:1 (1996), 75-82.

М. А. Скрябин (М. А. Skryabin)

Российский университет дружбы народов

E-mail: skryabinm@gmail.com
Представлено В. М. Тихомировым Принято редколлегией 28.10 .2008 